\section{Endometriumkarzinom: Auch Risikotypen lassen sich minimalinvasiv operieren}

\author{
Für Patientinnen, die an Hochrisiko-Subtypen von Endometriumkarzinomen \\ erkrankt sind, ist auch die minimalinvasive Chirurgie (MIC) eine Behandlungs- \\ option, wie eine retrospektive US-Studie nahelegt.
}

n die Untersuchung sind die Daten von 383 Patientinnen eingeflossen, die sich an einem von sieben US-amerikanischen Krebszentren wegen eines Endometriumkarzinoms Typ II oder Grad 3 einem operativen Staging unterziehen mussten. 191 wurden laparotomiert, 192 minimalinvasiv operiert (65\% per Roboter, $35 \%$ laparoskopisch). Klinisch befanden sich die Tumoren im Stadium I oder II. Die Primärtherapie erfolgte in Form einer Hysterektomie, SalpingoOophorektomie und pelvinen und aortalen Lymphonodektomie mit oder ohne Omentektomie.

Minimalinvasiv ließen sich im Median signifikant mehr Lymphknoten entfernen (39 vs. 34). Obwohl die SchnittNaht-Zeit bei MIC fast eine Stunde länger war als bei offenem Vorgehen (193 vs. 135 Minuten), konnten die Patientinnen die Klinik drei Tage früher verlassen (Aufenthalt 1 vs. 4 Tage). Dazu lag die Rate an Komplikationen - wie Infektionen, Ileus, nötige Transfusionen - nach MIC deutlich unter jener nach Laparotomie $(8,4 \%$ vs. $31,3 \%)$.

Die mediane Nachbeobachtungszeit umfasste 44 Monate. Die Rezidivrate betrug nach Laparotomie $26,1 \%$ und nach MIC 27,2\%, ein statistisch nicht signifikanter Unterschied. Aufgeschlüsselt nach Stadium und Vorgehensweise ergaben sich folgende Raten:

_Stadium I/II: MIC 17,3\%, Laparotomie $15,7 \%$;

—Stadium III/IV: MIC 51,8\%, Laparotomie $50,0 \%$.

Die meisten Rezidive fanden sich im extrapelvinen Raum (MIC: 74,2 \%; Laparotomie: $79,7 \%)$.

$83,6 \%$ der minimalinvasiv Operierten und $81,5 \%$ der Laparotomierten im Frühstadium überlebten die ersten drei Jahre nach dem Eingriff progressionsfrei. Für Patientinnen in späteren Krankheitsstadien lagen die Anteile bei $57,4 \%$ und 59,5\%. Die Anteile für das dreijährige Gesamtüberleben: $84,1 \%$ und 85,5\% (Frühstadium) sowie 63,8\% und $65,2 \%$ (Spätstadium). Wie bei den Rezidiv- zeigten sich auch bei den Überlebensraten keine signifikanten Unterschiede.

Fazit: Die Ergebnisse der vorliegenden Studie sprechen dafür, dass auch Hochrisiko-Typen von Endometriumkarzinomen minimalinvasiv operiert werden können - zumindest in klinisch frühen Stadien und an Zentren mit großer Erfahrung. Allerdings war die Untersuchung retrospektiv angelegt: Vorsicht also bei der Interpretation dieser Befunde, denn prospektive Daten fehlen noch.

Robert Bublak

Fader AN et al. Minimally invasive surgery versus laparotomy in women with high grade endometrial cancer. Gynecol Oncol. 2012;126(2):180-5.

Kommentar von Prof. Mallmann: Es liegen bereits mehrere prospektive randomisierte Studien zum Vergleich von Laparotomie und laparoskopischer Therapie des Endometriumkarzinoms vor, in denen die bekannten Vorteile des laparoskopischen Vorgehens, die reduzierte perioperative Morbidität, der verkürzte Krankenhausaufenthalt und die verbesserte Lebensqualität beim laparoskopischen Vorgehen im Vergleich zur Laparotomie ohne negativen Einfluss auf die Prognose der Erkrankung dokumentiert werden konnten. Dies wird auch im Rahmen der vorliegenden retrospektiven Studie bestätigt.

„Beim operablen Endometriumkarzinom ist offenbar ein laparoskopisches Vorgehen einer Laparotomie onkologisch gleichwertig."

Ein laparoskopisches Vorgehen ist mit einer signifikant längeren Operationsdauer, aber auch einer signifikant höheren Zahl entfernter Lymphknoten und einer signifikant erniedrigten Komplikationsrate verbunden. Bemerkenswert ist, dass hier erst-

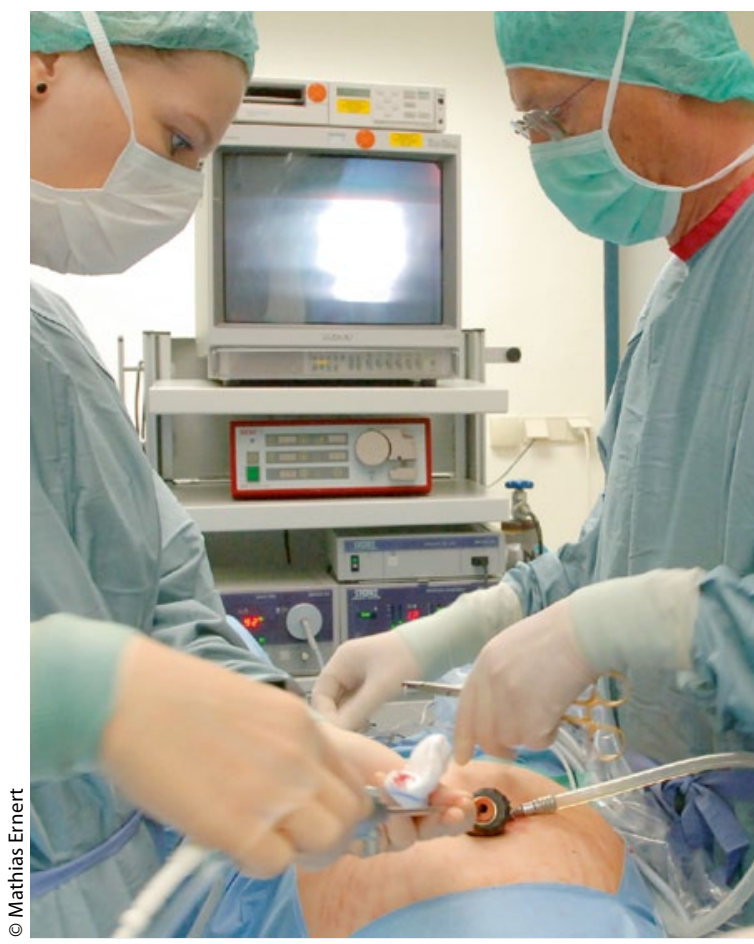

Die Vorteile eines laparoskopischen

Vorgehens möglichst breit nutzen.

malig auch ein roboterassistiertes laparoskopisches Vorgehen mit untersucht wurde, was keinen weiteren Vorteil im Vergleich zu einem rein laparoskopischen Vorgehen ergab. Ein Einfluss des Zugangsweges auf die Prognose fand sich nicht.

Während die derzeit gültigen S2-Leitlinien noch vergleichsweise zurückhaltend bezüglich des laparoskopischen Vorgehens sind („Bei Betrachtung der kurzfristigen postoperativen Morbidität ist das laparoskopische Vorgehen dem offen-chirurgischen Vorgehen überlegen. Bei Low-RiskFällen ist das onkologische Ergebnis wahrscheinlich gleichwertig"), zeigt diese retrospektive Analyse in Bestätigung der vorliegenden GOG-Studien nochmals, dass offenbar beim operablen Endometriumkarzinom ein laparoskopisches Vorgehen einer Laparotomie onkologisch gleichwertig ist.

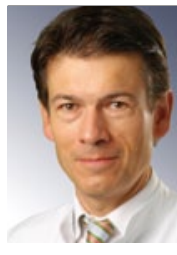

Prof. Dr. med. Peter Mallmann Klinik und Poliklinik für Frauenheilkunde und Geburtshilfe, Universität zu Köln, peter.mallmann@ uk-koeln.de 